\title{
Influência da urbanização sobre as assembléias de peixes em três córregos de Maringá, Paraná
}

\author{
Almir M. Cunico; Angelo A. Agostinho \& João D. Latini
}

Núcleo de Pesquisas em Ictiologia, Limnologia e Aqüicultura, Universidade Estadual de Maringá, Av. Colombo, 5790, Bloco H-90, 87020-900, Maringá, Paraná. E-mail: amcunico@brturbo.com.br

\begin{abstract}
Influence of urbanization upon fish assemblages in three streams of Maringá, Paraná. The aim of the present study was to evaluate spatial patterns in composition, structure and distribution of fish assemblages and their relationships with abiotic characteristics in three urban streams of Maringá city, Paraná, Brazil. Fish were collected along the longitudinal gradient by electrofishing in May and November 2004. Temperature, nitrogen, $\mathrm{pH}$, oxygen and phosphorous influenced at most the principal components analysis (PCA). Richness, evenness, Shannon-Wiener index and the first axis of detrended correspondence analysis (DCA) were directly correlated with abiotic characteristics related to chemical urban impact. Species addition prevailed in Miosotis stream sampling stations, and between headwater and intermediary reaches in Nazaré and Mandacarú streams. Replacement occurred between intermediary and lowland reaches of these streams. The dominance of the tolerant species Poecilia reticulata Peters, 1859 indicated the influence of the urban impact on fish assemblages. KEY WORDS. Chemical impact; Poecilia reticulata.
\end{abstract}

\begin{abstract}
RESUMO. O objetivo do presente estudo foi avaliar padrões espaciais na composição, estrutura e distribuição das assembléias de peixes e suas relações com características abióticas em três córregos urbanos da cidade de Maringá, Paraná, Brasil. Peixes foram coletados ao longo de um gradiente longitudinal utilizando pesca elétrica nos meses de maio e novembro de 2004. Temperatura, nitrogênio, pH, oxigênio e fósforo foram as variáveis que mais influenciaram a análise de componentes principais (CPA). Riqueza, eqüitabilidade, índice de diversidade de Shannon-Wiener e o primeiro eixo da análise de correspondência com remoção do efeito do arco (DCA) foram diretamente correlacionados com características ligadas ao impacto químico urbano. Adição de espécies prevaleceu nas estações de amostragem do córrego Miosótis e entre a cabeceira e porção intermediária nos córregos Nazaré e Mandacaru. Substituição ocorreu entre as porções intermediárias e foz desses córregos. A dominância da espécie tolerante Poecilia reticulata Peters, 1859 indicou a influência do impacto do meio urbano sobre as assembléias de peixes
\end{abstract}

PALAVRAS-CHAVES. Impacto químico; Poecilia reticulata.

Características físicas e químicas do hábitat têm sido reconhecidas como fatores de grande relevância na distribuição das espécies e na organização das comunidades em riachos (VANnote et al. 1980, Dyer et al. 1998). Para as assembléias de peixes, variações na composição, estrutura e distribuição das espécies têm sido associadas com mudanças na morfologia do canal (SCHLOSSER 1982), profundidade, tipo de substrato e corrente (Gorman \& KarR 1978, Angermeier \& KarR 1984), com a estruturação da vegetação marginal (JONEs III et al. 1999, BARRELA et al. 2001) e aspectos químicos da água, como concentrações de nutrientes, oxigênio dissolvido (Hughes 1987, Silva 1995, Honnen et al. 2001), substâncias cloradas (Osborne et al. 1981) e metais pesados (TuRnPenny \& Williams 1981).

Fortes evidências demonstram que as espécies exploram hábitats específicos, determinando padrões de distribuição característicos conforme as condições locais (GATZ 1979, UiedA 1984). Desta forma, alterações nas condições ambientais promovem uma reestruturação das assembléias ícticas, refletindo as condições vigentes da bacia hidrográfica em que estão inseridas (FAusch et al. 1990, Onorato et al. 1998).

Atualmente, a rápida expansão radial dos centros urbanos tem tido como conseqüência a degradação de habitats, com reflexos sobre os recursos naturais e nos ecossistemas aquáticos (LEE 2000). Embora a intensidade e duração de determinados fatores abióticos oscilem consideravelmente na natureza, ações antropogênicas têm geralmente amplificado seus efeitos, criando condições antes nunca encontradas pelas assembléias de peixes (EKLöv et al. 1998).

Revista Brasileira de Zoologia 23 (4): 1101-1110, dezembro 2006 
Poucos são os estudos que aval iam as respostas das espécies de peixes à urbanização (PAUL \& M AYER 2001), sendo este fato particularmente evidente na região neotropical, onde estes ambientes têm recebido menos atenção que os grandes rios (AGost।NHO \& JúLIO JR 1999). Esta carência de estudos explica a reduzida compreensão quetemos deste processo (LIMBURG \& ScHMIDT 1990). A falta de conhecimento, por outro lado dificulta severamente $o$ desenvolvimento de estratégias de manejo integradas, mitigação de impactos e políticas de conservação (MACDonneLL 1997).

Neste contexto, os objetivos deste trabal ho foram (I) avaliar os padrões espaciais na composição, estrutura e distribuição das assembléias ícticas e (II) relacionar estes padrões com características físicas e químicas do ambiente em três córregos urbanos da cidade de Maringá, visando avaliar a hipótese de que corpos d'água que apresentam suas nascentes inseridas no perímetro urbano possuem uma baixa riqueza específica, com a dominância de uma ou poucas espécies, ocorrendo alterações sobre a estrutura e distribuição das espécies ao longo de todo curso.

\section{MATERIAL E MÉTODOS}

\section{Área de estudo}

A bacia hidrográfica do rio Pirapó insere-se na região fisiográfica denominada Terceiro Planalto Paranaense, especificamente no polígono delimitado pelas latitudes de 22030' e 2330'S e longitudes de 51015' e 5215'W, com uma área de drenagem de aproximadamente $5076 \mathrm{~km}^{2}$ (SANEPAR 2002). A bacia é relativamente industrializada e urbanizada, sendo a cidade de Maringá o centro urbano mais importante da região, com cerca de 300 mil habitantes.

Devido à localização de M aringá sobre um espigão divisor de águas, vários cursos d água de dimensões reduzidas nascem na zona urbana, recebendo influência direta dos impactos antrópicos estabelecidos pelo meio urbano. Dentre os vários córregos que nascem na cidade foram escolhidos três para este estudo: córregos Mandacaru, Miosótis e Nazaré, sendo todos de primeira ordem, localizados na zona norte do município, e afluentes do ribeirão Maringá, um tributário do rio Pirapó, bacia do Paranapanema (Fig. 1). O rio Pirapó destaca-se por ser o rio responsável pelo abastecimento de água da cidade. As características qualitativas dos locais de amostragem estão descritas na tabela I.

\section{Coleta dos dados}

As coletas de peixes foram realizadas em maio e novembro de 2004, contemplando dois meses de el evada pluviosidade estando assim os ambientes amostrados mais suscetíveis a influência do despejo de água proveniente de galerias pluviais e da lixiviação superficial oriunda de superfícies impermeáveis do meio urbano.

Foi utilizado equipamento de pesca-elétrica (gerador portátil de corrente alternada 1 KW, 220V, 3-4 A) para as capturas, sendo amostrado os segmentos de cabeceira (próximos à nas-

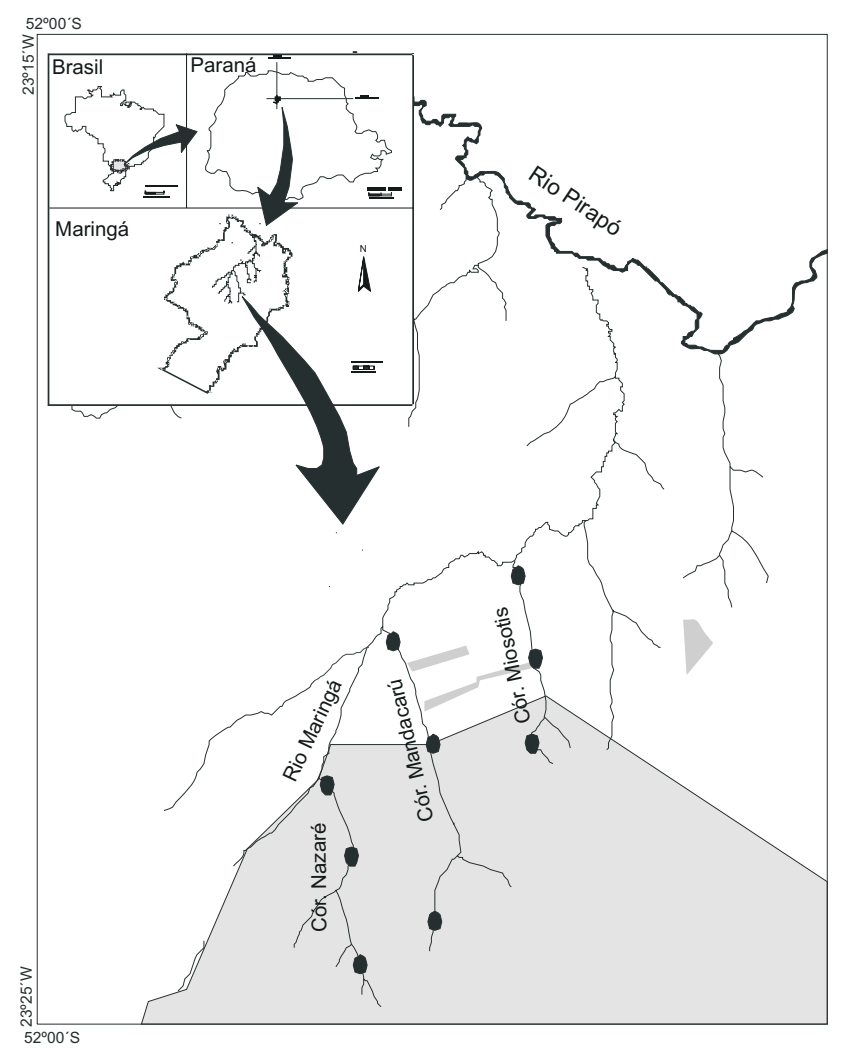

Figura 1. Localização das estações de amostragem nos três córregos analisados e a área de influência do meio urbano sobre os córregos (área em cinza).

cente), intermediário e foz (próximo à confluência com o ribeirão Maringá) de cada córrego, através de três capturas sucessivas com unidade de esforço constante (CPUE) sobre um trecho de $50 \mathrm{~m}$, ou menor extensão, quando aspectos físicos do ambiente não permitiram.

De cada segmento amostrado se estabeleceu medidas de área $\left(\mathrm{m}^{2}\right)$ e profundidade $(\mathrm{m})$, sendo aferida a partir de cinco transectos de 10 m de distância um do outro em cada segmento, com tomada de dados na margem direita, meio e margem esquerda dos córregos. As variáveis físico-químicas mensuradas em cada local foram a temperatura da água ( $\left({ }^{\circ} \mathrm{C}\right)$, condutividade elétrica $\left(\mu \mathrm{S} \mathrm{s}^{-1}\right.$ ), oxigênio dissolvido ( $\mathrm{mg} / \mathrm{l}$ e \% saturação), $\mathrm{pH}$, fluxo $(\mathrm{m} / \mathrm{s})$, concentrações de nitrogênio (ppm) e fósforo totais ( $\mathrm{mg} / \mathrm{L})$, sendo que as concentrações de nitrogênio e fósforo foram determinadas pelo laboratório de Limnologia do Núcleo de Pesquisas em Ictiologia, Limnologia e Aqüicultura, Universidade Estadual de Maringá.

Os peixes capturados foram preservados em formol $10 \%$, identificados, medidos (comprimento total e padrão), pesados e conservados em álcool $70 \%$. Os valores de densidade e biomassa de peixes foram determinados com base nas três remoções sucessivas em cada segmento amostrado e pela aplica-

Revista Brasileira de Zoologia 23 (4): 1101-1110, dezembro 2006 
Tabela I. Descrição qualitativa sucinta dos segmentos amostrados nos córregos Miosótis (Mi), Nazaré (Na) e Mandacaru (Ma). (Ca) Cabeceira, (In) intermediário, (Fo) Foz.

Pontos Descrição

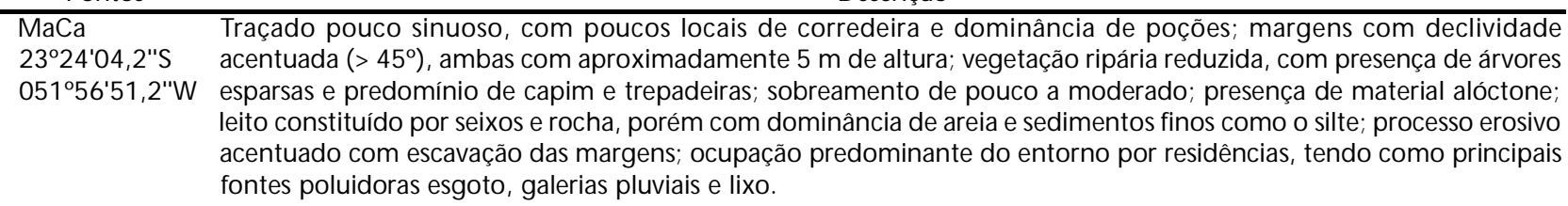

Maln Traçado pouco sinuoso, com predomínio de corredeiras e regiões de menor fluxo e maior profundidade; margens 232ㄱ'03,9"S com declividade pouco acentuada (<45ㅇ), ambas com aproximadamente 1,5 m; vegetação ripária ausente, com 051ำ56'47,5"W presença de gramíneas, arbustos e trepadeiras; sombreamento ausente; incipiente presença de material alóctone no leito fluvial; leito constituído por rochas, seixos e areia, porém com predominância de silte; processo erosivo acentuado; ocupação predominante do entorno por residências e pasto, tendo como principais fontes poluidoras galerias pluviais, esgoto, canalização e pisoteio marginal pelo gado.

Mafo Traçado moderadamente sinuoso, com predomínio de corredeiras; margens com declividade acentuada (>45o), 23021'28,8"S ambas com aproximadamente $3 \mathrm{~m}$ de altura; vegetação marginal reduzida, com árvores esparsas (eucalipto) porém 051으'15,9"W predomínio de capim; sombreamento moderado; pouca presença de material alóctone no leito fluvial; leito constituído de seixos e silte; processo erosivo acentuado com escavação das margens; ocupação predominante do entorno por plantações de soja e residências, tendo como principais fontes poluidoras pesticidas e adubos químicos, esgoto e galerias pluviais.

Traçado moderadamente sinuoso, com canal estreito apresentando locais de corredeira porém com presença de 23025'06,3"S poções; margens com declividade pouco acentuada (<45ㅇ), porém estendendo-se por mais de 20 m; margem direita 2325'06,3"S com aproximadamente 20 m de vegetação apresentando árvores esparsas e margem esquerda com vegetação quase 051-57'39,1"W ausente, porém com um extensa área onde se efetuou reflorestamento; ambiente sombreado; abundante presença de material alóctone no leito fluvial; leito constituído por seixos, cascalho e deposição de pedras e areia nos meandros; processo erosivo acentuado; ocupação predominante do entorno por residências, industrias metalúrgicas e uma avenida de grande fluxo de veículos tendo como principais fontes poluidoras galerias pluviais, dejetos industrias e recepção de água proveniente do escoamento de superfícies impermeáveis.

Naln Traçado pouco sinuoso, com locais de corredeira, remansose poções; margens com declividade pouco acentuada (< 2324'24,1"S 45) e aproximadamente 3 metros de altura; vegetação marginal praticamente ausente, constituída basicamente por 05157'54,6"W capim; ambiente pouco sombreado; presença irrisória de material alóctone no leito fluvial; leito constituído por seixos, algumas lajes rochosas e sedimentos finos oriundos das margens; processo erosivo acentuado; ocupação predominante do entorno por residências e plantio, tendo como principais fonte poluidoras, lixo, galerias pluviais, esgoto, adubos e pesticidas.

NaFo Traçado pouco sinuoso, com corredeiras fortes alternadas por poções profundos, margens com declividade acentuada 23ㄴ23'07,0"S (>45ㅇ) e aproximadamente $4 \mathrm{~m}$ de altura; vegetação marginal praticamente ausente, constituída basicamente por 05157'59,2"W capim; ambiente não sombreado; sem presença de material alóctone no leito fluvial; leito constituído por seixos, cascalho e barro; processo erosivo acentuado com escavação das margens; ocupação predominante do entorno por residências e plantio de hortaliças, tendo como principais fontes poluidoras, lixo, esgoto, galerias pluviais e recepção de água provenientes de superfícies impermeáveis.

MiCa Traçado moderadamente sinuoso, apresentando trechos de corredeira com pouca profundidade, alternado por 2322'22,6" S poções; margens com declividade acentuada, inclinação de aproximadamente 45으 5 m de altura; margem esquerda 0515ㄴ'43 1"W com árvores esparsas e grande densidade de cipóse trepadeiras e margem direita vegetada por capim; sombreamento moderado; presença de material alóctone no leito fluvial como troncos, galhos e folhas; processo erosivo presente de maneira acentuada com escavação das margens e inserção de sedimentos finos no leito fluvial; leito de fundo rochoso com acúmulo de sedimentos finos em regiões de menor correnteza; ocupação predominante do entorno por residências, pasto e plantio, tendo como principais fonte poluidora aparente despejo de água proveniente de galerias pluviais.

Miln Traçado pouco sinuoso e encaixado, apresentando trechos de corredeira com algumas regiões de remanso; margem 2321'59,3"S com declividade acentuada, inclinação superior a 45 e 5 m de altura; margens com algumas árvores, principalmente 05155'35,6"W eucaliptos, porém com predominância de capim; sombreamento moderado; presença de material alóctone no leito fluvial como troncos, galhos e folhas, processo erosivo presente de maneira acentuada, com escavação profunda das margens e inserção de sedimentos finos no leito fluvial, leito constituído por seixos e elevada concentração de sedimento finos elevando a turbidez; ocupação predominante do entorno por residências e plantio de soja, tendo como principais fontes poluidoras possivelmente pesticidas e adubos químicos.

MiFo Traçado pouco sinuoso e encaixado, com predomínio de trechos de corredeira; margem esquerda com declividade 2320'38,6"S acentuada (> 45) e direita pouco íngreme (<45ㅇ), ambas com aproximadamente 5 metros de altura; vegetação 051ㅇ5'51,0"W marginal ausente em todo o trecho amostrado, constituída basicamente por capim; sombreamento ausente; ausência de material alóctone no leito fluvial; leito constituído por seixos e grande concentração de sedimentos finos oriundos da erosão marginal; processo erosivo acentuado com escavação das margens e retirada de meandros; ocupação predominante do entorno por plantio de soja (principalmente) e pasto, tendo como principais fontes poluidoras possivelmente pesticidas e adubos químicos. 
ção do método de ZIPPIN (1958), sendo os resultados expresso em número de indivíduos e quilogramas por hectare.

Análise dos dados

Avaliou-se a estrutura das assembléias através dos atributosRiqueza (S), representando o número de espécies; Eqüitabilidade (E), indicando a distribuição da abundância entre as espécies, e obtido através da seguinte equação: $E=H^{\prime} / \operatorname{logS}$, onde $\mathrm{H}^{\prime}$ = índice de diversidade de Shannon e $\mathrm{S}=$ riqueza (Pielou 1975); e Diversidade ( $\left.H^{\prime}\right)$, índice que considera a riqueza e a eqüitabilidade das espécies, sendo calculado através do índice de Shannon-Wiener (MAGURRAN 1988), dado pela equação: $\mathrm{H}^{\prime}=-\Sigma$ pi.In.pi, onde In = logaritmo natural e pi = proporção de indivíduos na amostra.

Alterações na composição específica ao longo de um gradiente longitudinal foram avaliadas pelo índice de diversidade Beta-2 ( $\beta$-2) (HARRISON et al. 1992), calculado entre cada estação de amostragem pela seguinte fórmula:

Beta $-2=\frac{\frac{S}{\alpha \max }-1}{N-1} \times 100$ onde, S é o número total de espécies em ambas as estações de amostragem; $\alpha$ max é o máximo número de espécies encontrado em uma das estação de amostragem e $\mathrm{N}$ é o número de estações de amostragem.

Os padrões da estrutura da comunidade íctica foram analisados utilizando análise de correspondência com remoção do efeito do arco (DCA), com os dados de densidade específica, e as variáveis abióticas sumarizadas pela análise de componentes principais (PCA) utilizando o programa PC-ORD 3.0 (MCCUNE \& MefFORd 1997). Para determinar quais componentes principais seriam retidos para análise foi utilizada a hipótese de aleatoriedade obtida do modelo de "broken-stick" (JACKSON 1993). Todos os dados limnológicos, com exceção do pH, foram $\log _{10}(x+1)$ transformados previamente à análise.

Correlações de Pearson foram feitas entre os escores dos eixos da PCA e os valores de riqueza, eqüitabilidade, diversidade, densidade específica e os escores dos eixos da DCA com o intuito de investigar a potencial influência das variáveis abióticas nos atributos e na estrutura das assembléias.

\section{RESULTADOS}

\section{Características físico-químicas}

Os três córregos analisados percorrem diferentes extensões dentro do perímetro urbano (Fig. 1), apresentando alterações em suas características fisionômicas ao longo do curso. A análise de componentes principais (PCA) explicou nos dois primeiros eixos $64,5 \%$ da variabilidade total dos dados, sendo estes eixos retidos para interpretação. Temperatura da água, nitrogênio total e fluxo foram correlacionados positivamente com o eixo 1, enquanto o pH e a condutividade correlacionaram-se negativamente. Oxigênio dissolvido, porcentagem de saturação de oxigênio e profundidade apresentaram correla- ção positiva, enquanto a concentração de fósforo total foi ne gativamente correlacionada com o eixo 2 (Tab. II).

Tabela II. Resultado da análise de componentes principais (PCA). Os coeficientes foram obtidos usando correlação de Pearson.

\begin{tabular}{lrr}
\hline & PC1 & \multicolumn{1}{c}{ PC2 } \\
\hline Autovalores & 2,978 & 2,827 \\
\% de explicação & 33,084 & 31,416 \\
Temperatura da água & 0,770 & $-0,225$ \\
pH & $-0,781$ & 0,212 \\
Condutividade & $-0,662$ & 0,354 \\
O2 & 0,204 & 0,825 \\
\% Saturação O2 & 0,552 & 0,635 \\
Nitrogênio & 0,681 & $-0,483$ \\
Fósforo & $-0,154$ & $-0,709$ \\
Fluxo & 0,614 & 0,606 \\
Profundidade & 0,355 & 0,648 \\
\hline
\end{tabular}

Analisando a ordenação (Fig. 2), nota-se no segundo eixo tendência no estabelecimento de um gradiente longitudinal, no qual as porções de cabeceira possuem maior concentração de fósforo e menor concentração de oxigênio dissolvido, porcentagem de saturação de oxigênio e profundidade.

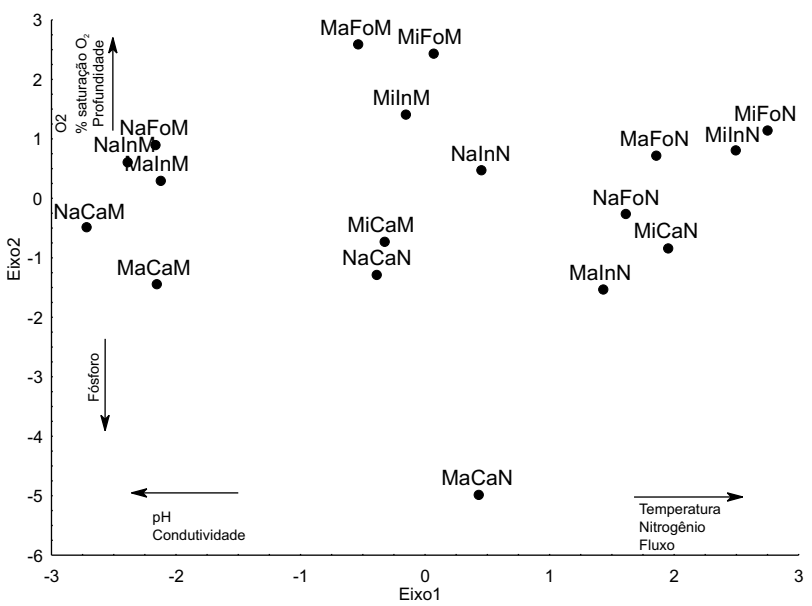

Figura 2. Análise de Componentes Principais entre as variáveis físicas e químicas dos locais de coleta. (Ma) Mandacaru, (Na) Nazaré, (Mi) Miosótis, (Ca) Cabeceira, (In) Intermediário, (Fo) Foz, (M) maio, (N) novembro.

\section{Ictiofauna}

Foram coletados 7.987 exemplares, pertencentes a seis ordens, sete famílias, 10 gêneros e 11 espécies, perfazendo uma biomassa total de 14,37 Kg. Dentre as espécies, 83\% pertence- 
ram a espécie Poecilia reticulata Peters, 1859 e 16\% à espécies da ordem Siluriformes, sendo que a soma das demais ordens não atingiu $1 \%$ do total coletado.

Apenas 10 exemplares (cerca 0,13\% do total) apresentaram comprimento padrão acima de $150 \mathrm{~mm}$ (Tab. III). No córrego Mandacaru, das seis espécies presentes nas amostragens três (Poecilia reticulata, Poeciliidae; Rhamdia quelen (Quoy \& Gaimard, 1824), Heptapteridae e Hypostomus ancistroides (Ihering, 1911), Hypostominae) foram encontradas em todos os segmentos amostrados; no córrego Miosótis cinco das oito espécies foram coletadas em todos os segmentos (Cetopsorhamdia iheringi Schubart \& Gomes, 1959, Heptapteridae; Imparfinis schubarti (Gomes, 1956), Heptapteridae; P. reticulata; R. quelen e H. ancistroides) e no córrego Nazaré, das seis espécies amostradas, apenas $\mathrm{P}$. reticulata esteve presente em todos os segmentos.

As três espécies mais representativas em abundância somaram 98\% da densidade total, sendo elas: P. reticulata (83\%), H. ancistroides $(10,8 \%)$ e R. quelen $(4,2 \%)$ e $76,2 \%$ da biomassa total: R. quelen (41\%) e H. ancistroides (35,2\%). Poecilia reticulata foi dominante nas porções de cabeceira, apresentando porém abundância rel evante em todos os segmentos amostrados. Apenas nas porções inferiores do córrego Miosótis essa espécie não esteve entre as três mais representativas (Tab. III).

Com relação aos atributos de comunidade, a cabeceira do córrego Nazaré apresentou a menor riqueza, com apenas uma espécie (P. reticulata), e a foz do córrego Miosótis os maiores valores de riqueza, eqüitabilidade e diversidade de ShannonWiener, (Tab. IV).

$O$ índice de diversidade $\beta-2$ indicou processo de adição e substituição de espécies ao longo dos córregos. Valores do índice iguais a zero foram encontrados entre os segmentos superiores, indicando a adição de espécies nestes trechos (cabeceiraintermediário). Já para os segmentos inferiores dos córregos Mandacaru e Nazaré foram caracterizados o processo de substituição específica ocorrendo a maior heterogeneidade entre o segmento intermediário e a foz do córrego Mandacaru ( $\beta-2=$ $0,20)$, seguido do córrego Nazaré $(\beta-2=0,16)$. No córrego Miosótis ocorreu o processo de adição com a presença de duas novas espécies na porção inferior.

O padrão de distribuição longitudinal estabelecido para

Tabela III. Densidade, biomassa e comprimento padrão das espécies capturadas nos três segmentos amostrados nos córrego.

\begin{tabular}{|c|c|c|c|c|c|c|c|c|c|}
\hline \multirow{2}{*}{ Locais/espécies } & \multicolumn{3}{|c|}{ Densidade $\left(\mathrm{N} \cdot \mathrm{ha}^{-1}\right)$} & \multicolumn{3}{|c|}{ Biomassa $\left(\mathrm{Kg}_{\mathrm{h}} \mathrm{ha}^{-1}\right)$} & \multicolumn{3}{|c|}{ Comprimento padrão (cm) } \\
\hline & Cab & Inte & Foz & Cab & Inte & Foz & Cab & Inte & Foz \\
\hline \multicolumn{10}{|l|}{ Mandacaru } \\
\hline A. paranae & 71,16 & 418,95 & - & 1,124 & 0,703 & - & 7,30 & $3,00-5,0$ & - \\
\hline G. carapo & - & - & 73,26 & - & - & 0,858 & - & - & 2,6 \\
\hline H. ancistroides & 967,58 & 33988,64 & 7946,00 & 7,164 & 100,934 & 30,020 & $2,00-11,50$ & $2,00-12,5$ & $1,5-12,0$ \\
\hline P. reticulata & 337215,67 & 59753,75 & 5545,95 & 113,480 & 38,193 & 2,243 & $0,02-2,47$ & $1,20-4,2$ & $1,0-4,2$ \\
\hline R. quelen & 5319,48 & 4468,70 & 1912,04 & 144,065 & 54,563 & 44,108 & $5,00-16,50$ & $3,70-13,0$ & $4,4-15,5$ \\
\hline S. marmoratus & - & 47,06 & - & - & 1,255 & - & - & 17,50 & - \\
\hline \multicolumn{10}{|l|}{ Miosótis } \\
\hline A. paranae & - & 133,30 & 69,68 & - & 0,764 & 0,087 & - & $4,00-6,4$ & 3,0 \\
\hline C. iheringi & 56,49 & 622,90 & 2157,57 & 1,093 & 4,435 & 8,277 & - & $6,40-8,3$ & $4,0-8,4$ \\
\hline G. brasiliensis & - & - & 69,68 & - & - & 1,002 & - & - & 7,0 \\
\hline G. carapo & - & - & 108,69 & - & - & 0,141 & - & - & 6,2 \\
\hline H. ancistroides & 2610,35 & 5258,58 & 8975,58 & 43,531 & 31,592 & 40,613 & $3,90-16,50$ & $3,00-8,8$ & $1,5-9,0$ \\
\hline I. schubarti & 123,06 & 935,70 & 4997,02 & 0,367 & 6,238 & 8,084 & $5,40-5,50$ & $1,30-6,0$ & $2,2-6,9$ \\
\hline P. reticulata & 21271,34 & 997,62 & 1255,26 & 17,505 & 0,491 & 0,721 & $0,80-\quad 3,90$ & $1,10-4,0$ & $1,5-3,0$ \\
\hline R. quelen & 5334,01 & 6167,82 & 2710,84 & 140,217 & 75,469 & 39,944 & $3,90-16,50$ & $4,60-13,5$ & $3,4-13,0$ \\
\hline \multicolumn{10}{|l|}{ Nazaré } \\
\hline A. paranae & - & 65,46 & - & - & 0,734 & - & - & 7,00 & - \\
\hline C. iheringi & - & & 2754,20 & - & - & 22,285 & - & - & $3,8-8,5$ \\
\hline H. ancistroides & - & 2785,20 & 13707,67 & - & 29,019 & 34,691 & - & $2,10-8,5$ & $2,0-9,5$ \\
\hline H. strigaticies & - & - & 121,20 & - & - & 0,257 & - & - & $4,2-4,5$ \\
\hline P. reticulata & 220674,20 & 5965,81 & 29866,72 & 5,418 & 3,413 & 14,014 & $0,70-4,50$ & $1,30-3,9$ & $1,2-3,7$ \\
\hline P. tenebrosa & - & - & 326,25 & - & - & 0,814 & - & - & $5,5-8,0$ \\
\hline R. quelen & - & 2348,17 & 602,41 & - & 62,487 & 3,652 & - & 1465,95 - & $5,5-8,5$ \\
\hline
\end{tabular}


Tabela IV. Valores de riqueza, eqüitabilidade e índice de diversidade de Shannon-Wiener de todos os segmentos amostrados nos três córregos analisados no estudo. ( $\mathrm{Ma}$ ) Mandacaru, ( $\mathrm{Na}$ ) Nazaré, (Mi) Miosótis, (Ca) Cabeceira, (In) Intermediário, (Fo) Foz, (M) maio, (N) novembro.

\begin{tabular}{llll}
\hline \multicolumn{1}{c}{ Name } & $\mathrm{S}$ & \multicolumn{1}{c}{$\mathrm{E}$} & $\mathrm{H}^{\prime}$ \\
\hline MiCaM & 3 & 0,724 & 0,795 \\
MilnM & 5 & 0,851 & 1,370 \\
MiFoM & 6 & 0,857 & 1,536 \\
MiCaN & 5 & 0,468 & 0,754 \\
MilnN & 5 & 0,742 & 1,194 \\
MiFoN & 7 & 0,670 & 1,303 \\
NaCaM & 1 & 0 & 0 \\
NalnM & 4 & 0,668 & 0,925 \\
NaFoM & 5 & 0,489 & 0,788 \\
NaCaN & 1 & 0 & 0 \\
NalnN & 3 & 0,794 & 0,873 \\
NaFoN & 5 & 0,678 & 1,091 \\
MaCaM & 4 & 0,080 & 0,111 \\
MalnM & 4 & 0,589 & 0,816 \\
MaFoM & 4 & 0,686 & 0,951 \\
MaCaN & 3 & 0,033 & 0,036 \\
MalnN & 4 & 0,594 & 0,823 \\
MaFoN & 3 & 0,658 & 0,723 \\
\hline
\end{tabular}

os dados abióticos não se apresentou de maneira evidente com relação à estrutura da comunidade íctica. A análise de correspondência com remoção do efeito do arco (DCA) aplicada aos dados de densidade específica teve apenas o primeiro eixo retido para interpretação (eixo $1=0,52$; eixo $2=0,06$ ) não se estabelecendo um padrão nítido de distribuição longitudinal, porém com tendência de separação entre a porção superior (cabeceira) e inferior (intermediário e foz) dos córregos (Fig. 3).

Correlações significativas entre o eixo 2 da PCA e o eixo 1 da DCA (Fig. 4) e entre o eixo 2 da PCA e os atributos da comunidade (Fig. 5) sugeriram uma forte relação entre as variáveis abióticas (concentração de fósforo, oxigênio dissolvido, porcentagem de saturação de oxigênio e profundidade), os atributos da comunidade (riqueza específica, eqüitabilidade, índice de diversidade de Shannon-Wiener) e a distribuição espacial das espécies. Altos valores de riqueza, eqüitabilidade e diversidade de Shannon-Wiener estiveram associados com redução na concentração de fósforo e aumento dos valores de oxigênio dissolvido, porcentagem de saturação de oxigênio e profundidade.

A correlação de Pearson estabelecida entre a densidade de Poecilia reticulata e o eixo dois da PCA apresentou correlação negativa, revelando maior abundância em locais com maior concentração de fósforo e menor concentração de oxigênio dissolvido, porcentagem de saturação de oxigênio e profundidade, com tendência à redução de sua abundância em direção à foz dos córregos amostrados (Fig. 6).
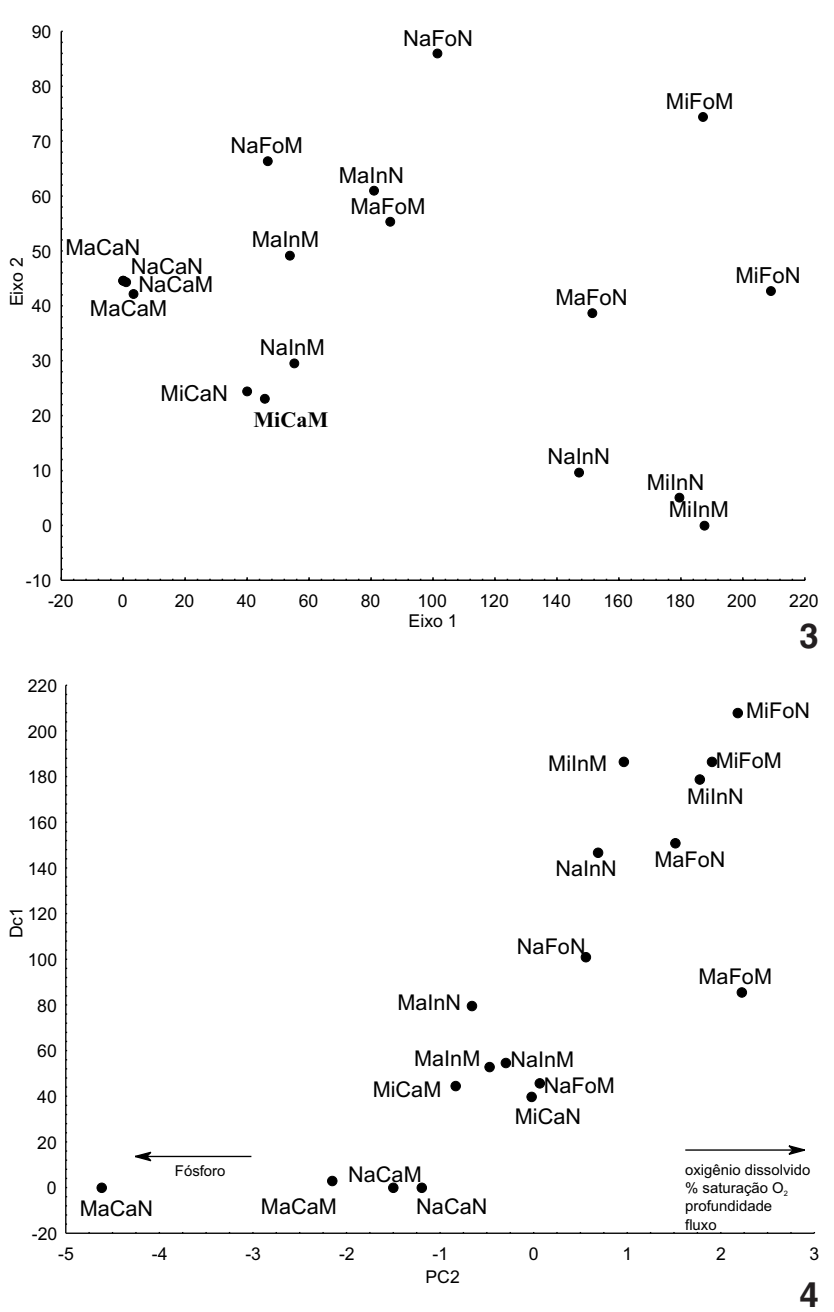

Figuras 3-4. (3) Análise de Correspondência com remoção do efeito do arco (DCA) para os dados de densidade específica, mostrado a distribuição espacial das espécies nos diferentes segmentos amostrados; (4) correlações entre os escores do eixo 2 da PCA e os escores do eixo 1 da DCA. (Ma) Mandacaru, (Na) Nazaré, (Mi) Miosótis, (Ca) Cabeceira, (In) Intermediário, (Fo) Foz, (M) maio, (N) novembro.

\section{DISCUSSÃO}

Numerosos estudos evidenciam a influência de alterações nas características físicas e químicas do hábitat sobre o padrão de distribuição das espécies de peixes, destacando um aumento na diversidade específica ao longo de um gradiente longitudinal (Kuenne 1962, Sheldon 1968). Entretanto, a presença de poluentes e demais fontes de impactos provenientes de atividades antrópicas proporcionam a redução do número de espécies e o aumento da densidade de espécies resistentes às variações ambientais (REASH \& BerRA 1987). M ARgalef (1963) destaca que ambientes com fortes flutuações ambientais permanecem 

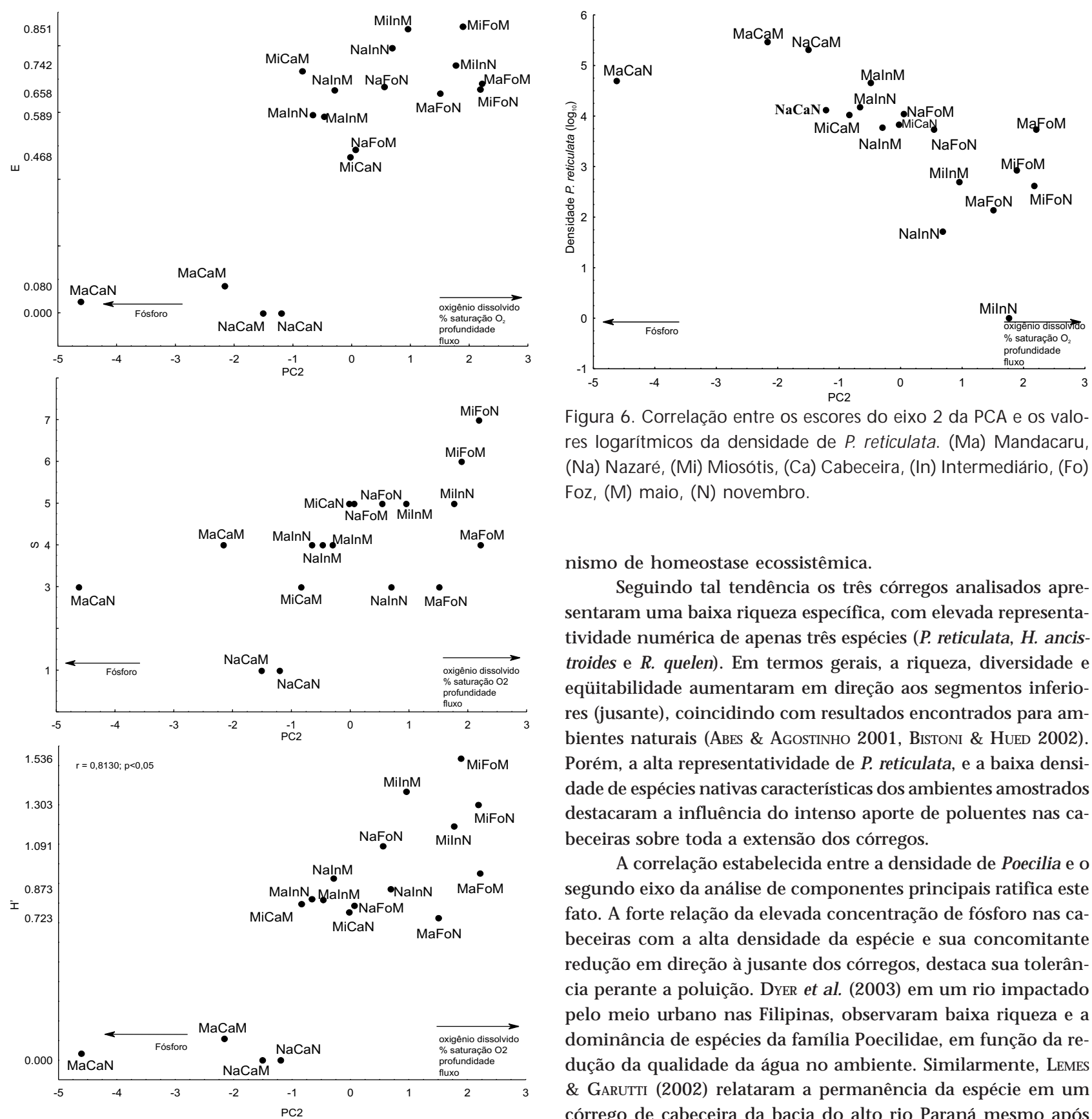

Figura 6. Correlação entre os escores do eixo 2 da PCA e os valores logarítmicos da densidade de $\mathrm{P}$. reticulata. (Ma) Mandacaru, (Na) Nazaré, (Mi) Miosótis, (Ca) Cabeceira, (In) Intermediário, (Fo) Foz, (M) maio, (N) novembro.

nismo de homeostase ecossistêmica.

Seguindo tal tendência os três córregos analisados apresentaram uma baixa riqueza específica, com elevada representatividade numérica de apenas três espécies (P. reticulata, $\mathrm{H}$. ancistroides e R. quelen). Em termos gerais, a riqueza, diversidade e eqüitabilidade aumentaram em direção aos segmentos inferiores (jusante), coincidindo com resultados encontrados para ambientes naturais (Abes \& Agostinho 2001, Bistoni \& Hued 2002). Porém, a alta representatividade de P. reticulata, e a baixa densidade de espécies nativas características dos ambientes amostrados destacaram a influência do intenso aporte de poluentes nas cabeceiras sobre toda a extensão dos córregos.

A correl ação estabel ecida entre a densidade de Poecilia e o segundo eixo da análise de componentes principais ratifica este fato. A forte relação da el evada concentração de fósforo nas cabeceiras com a alta densidade da espécie e sua concomitante redução em direção à jusante dos córregos, destaca sua tolerância perante a poluição. DYER et al. (2003) em um rio impactado pelo meio urbano nas Filipinas, observaram baixa riqueza e a dominância de espécies da família Poecilidae, em função da redução da qualidade da água no ambiente. Similarmente, LEMES \& GARUTTI (2002) relataram a permanência da espécie em um córrego de cabeceira da bacia do alto rio Paraná mesmo após um derramamento de óleo queimado, evidenciando sua grande plasticidade perante drásticas alterações ambientais.

O aporte de fósforo nos corpos d'água analisados possieqüitabilidade (E), riqueza (S) e índice de diversidade de Shanon $\left(\mathrm{H}^{\prime}\right)$. (Ma) Mandacaru, ( $\left.\mathrm{Na}\right)$ Nazaré, (Mi) Miosótis, (Ca) Cabeceira, (In) Intermediário, (Fo) Foz, (M) maio, (N) novembro.

em um estado de baixa maturidade, onde espécies com maior taxa potencial de crescimento tornam-se dominantes, reduzindo a diversidade e desta forma dificultando um eficiente mecavelmente ocorre em função do despejo clandestino de esgoto por ligações irregulares, descarga de caminhões auto-fossa e lixiviação superficial através de redes de galerias pluviais (observação in loco). Segundo PAUL \& MEYER (2001) a elevação nas concentrações de nutrientes no meio urbano, principalmente fósforo total, ocorre devido ao aumento da inserção de partí- 
culas associadas ao elemento presente em esgotos, bem como da lixiviação de superfícies impermeáveis, estabelecendo redução dos níveis de oxigênio e promovendo alterações sobre a composição e estrutura da ictiofauna (Bö̈t et al. 1999).

A baixa ocorrência e densidade de indivíduos da ordem Characiformes, especialmente da família Characidae, também evidenciou efeitos da poluição urbana sobre a estrutura das assembléias dos córregos. M esmo a família Characidae não sendo exclusiva de riachos, seus membros geralmente formam o principal conjunto de espécies de meia água nestes ambientes (BucKuP 1999). Penczak et al. (1994), Pavanelli \& Caramaschi (1997) e CasTRO \& CASATTI (1997) verificaram em seus estudos em riachos na bacia do alto Rio Paraná, a presença de espécies constituintes desta família (Astyanax spp.) entre as mais abundantes nos locais estudados. Entretanto, mesmo tendo conhecimento de que tais espécies possuem uma grande flexibilidade em ocupar ambientes diferenciados e apresentam estratégias para isso, locais com melhores condições, mais preservados e próximos da situação original possibilitam às espécies explorar de forma mais eficiente e abrangente, com reflexos positivos na estrutura populacional (OrsI et al. 2004).

A presença de distúrbios e a dominância de espécies tolerantes em detrimento das mais sensíveis estabelecem marcantes alterações na estrutura e distribuição da fauna de peixes (JACKSON et al. 2001). Nos ambientes analisados se evidenciaram tais alterações, ocorrendo tendência de separação entre as porções superiores e inferiores com relação à estruturação das assembléias. Apenas a cabeceira do córrego Miosótis teve leve distinção da cabeceira dos demais córregos, possivel mente em virtude de percorrer menor extensão inserida no meio urbano e de possuir menor concentração residencial no entorno, proporcionando assim condições ambientais mais favoráveis.

A análise de componentes principais e a correlação de Pearson destacaram variação na riqueza, eqüitabilidade e diversidade, relacionadas com aspectos químicos da água, podendose assim estabelecer o aporte de poluentes orgânicos como possível fator de impacto sobre a ictiofauna. Os córregos de Maringá demonstram elevado grau de poluição devido à ocupação urbana (loteamentos e indústrias), às atividades agropecuárias desenvolvidas nas microbacias e ao recebimento de efluentes diversos como esgoto doméstico não tratado. BoËt et al. (1999), GAFNY et al. (2000) e GISPERT et al. (2002) estabeleceram relações similares entre o elevado aporte de matéria orgânica em zonas urbanas, al terações nas condições químicas da água e na integridade da fauna íctica. Chuvas torrenciais, as quais carreiam grandes concentrações de matéria orgânica e lixo de superfícies impermeáveis, foram associadas com a mortalidade de peixes no rio Sena, dentro do perímetro urbano de Paris (Bö̈T et al. 1999), um efeito também observado no rio Yargon, o maior rio urbano de Israel (GAfNy et al. 2000).

Embora ocorrendo tendência de melhora das condições químicas e biológicas nas porções inferiores dos córregos, a alta representatividade numérica de apenas três espécies em todos os locais amostrados estabel eceu evidências de que a degradação química não se restringiu apenas à porção urbanizada, mas também às regiões adjacentes. DYER et al. (2003) relataram reduzida riqueza e dominância específica em toda a extensão fluvial, mesmo estando a entrada de poluentes restrita a um único ponto altamente urbanizado. Desta maneira, as porções inferiores não estão livres dos impactos oriundos da urbanização e da ação sinérgica com os impactos locais.

Os aumentos nos val ores de riqueza, eqüitabilidade e diversidade em direção à foz dos córregos ocorreram em função da presença de espécies raras nas coletas, como Synbranchus marmoratus Bloch, 1795, Synbranchidae; Gymnotus carapo Linnaeus, 1758, Gymnotidae; Astyanax paranae Eigenmann, 1914, Characidae; Hypostomus strigaticipes (Regan, 1908), Hypostominae; Geophagus brasiliensis (Quoy \& Gaimard, 1824), Cichlidae e Phenacorhamdia tenebrosa (Schubart, 1964), Heptapteridae, sendo que as espécies mais representativas numericamente apresentaram-se dominantes em todos os locais amostrados, oscilando apenas o "ranking" de dominância. Em rios tributários da bacia do rio São Franscisco, EUA, onde a fauna nativa de peixes é depauperada, LeIdy \& FIELDER (1985) encontraram que tanto a riqueza de espécies como a diversidade, aumentam nos locais sujeitos a um moderado distúrbio humano. Segundo FAusch et al. (1990), os atributos de comunidade declinam com a severa degradação, porém a riqueza de espécies e a diversidade tendem a aumentar mesmo em locais com menor ou moderada degradação.

O índice de diversidade Beta-2, da mesma forma, evidenciou aumento da heterogeneidade ambiental e da ictiofauna em direção à jusante, corroborando com estudos prévios para ambientes naturais (PALLeR 1994, BISTONI \& HUED 2002). Entretanto, a manutenção da alta representatividade de espécies tolerantes à poluição e a não substituição destas por espécies características dos segmentos inferiores evidenciou, embora não de maneira exclusiva, a manutenção de estresses presentes nas porções de cabeceira sobre toda a extensão dos córregos. Segundo TRAMER \& ROGERs (1973), variações na qualidade da água desordenam o padrão normal de zonação longitudinal das espécies, apresentando a composição específica de todo o sistema níveis similares ao das cabeceiras.

As assembléias de peixes em pequenos rios (primeira até terceira ordem) estão particularmente em risco devido a impactos oriundos do meio urbano. Rios de pequena ordem exibem naturalmente baixa riqueza específica sendo altamente suscetíveis a perda de espécie e redução da diversidade por mudanças induzidas pela urbanização na qualidade da água, regime hidrológico ou ambos. Logo sem o conhecimento de quais espécies vivem nestes sistemas, como elas interagem dentro de ambientes aquáticos altamente modificados ou como a biota responde a urbanização e seus impactos específicos, nós estaremos despreparados para estabelecer efetivas estratégias de conservação em ecossistemas aquáticos urbanos. 


\section{AGRADECIMENTOS}

Ao Núcleo de Pesquisas em Limnologia, Ictiologia e Aqüicultura (NUPÉLIA) da Universidade Estadual de Maringá. Ao CNPq pela concessão da bolsa ao primeiro autor. A W.J. da Graça e C.H. Zawadzki pela identificação das espécies. A R.M. Buchas e C.S. Pavanelli pelas valiosas sugestões.

\section{REFERÊNCIAS BIBLIOGRÁFICAS}

AbEs, S.S. \& A.A. Agostinho. 2001. Spatial patterns in fish distribuitions and structure of the ichthyocenosis in the Água Nanci stream, upper Paraná River basin, Brazil. Hydrobiologia, Dordrecht, 445: 217-227.

Agostinho, A.A. \& H.F. Júlio JR. 1999. Informar a referência completa seguindo as Instruções aos Autores ou suprimir a chamada do texto.

Angermeier, P.L. \& J.R. KARR. 1984. Relationships between Woody Debris and Fish Hábitat in a Small Warmwater Stream. Transactions of the American Fisheries Society, Bethesda, 113: 727-736.

Barrela, W.; M. Petrere Júnior; W.S. Smith \& L.F.A. M ontag. 2001. As relações entre matas ciliares, os rios e os peixes, p. 187208. In: R.R. Rodrigues \& H.F. LeitÃo Filho (Ed.). Matas ciliares: conservação e recuperação. São Paulo, EDUSP, 320p.

Bistoni, M.A. \& A.C. Hued. 2002. Patterns of fish species richness in rivers of the central region of Argentina. Brazilian Journal of Biology, Rio de Janeiro, 62 (4B): 753-764.

Bö̈t, P.; J. Belliard; R. BerReBI-DIT-Thomas \& E. Tales. 1999. Multiple human impacts by the city of Paris on fish communities in the Seineriver basin, France. Hydrobiologia, Dordrecht, 410: 59-68.

Buckup, P. A. 1999. Sistemática e biogeografia de peixes de riachos, p. 91-135. In: E. P. CARAMASChI; R. MazzonI; C.R.S.F. Bizerril \& P.R. Peres-Neto (Eds). Ecologia de peixes de riacho: estado atual e perspectivas. Rio de Janeiro, Oecologia Brasiliensis, 260p.

CASTRO, R.M.C. \& L. CASATtI. 1997. The fish fauna from a small forest stream of the upper Paraná river basin, southeastern Brazil. Ichthyological Exploration of Freshwaters, München, 7 (4): 337-352.

Dyer, S.D.; C.E. White-Hull; X. Wang; T.D. Johnson \& G.J. CarR. 1998. Determining the influence of habitat and chemical factors on instream biotic integrity for a Southern Ohio watershed. Journal of Aquatic Ecosystem Stress and Recovery, Dordrecht, 6: 91-110.

Dyer, S.D.; C. Peng; D.C. McAvoy; N.J. Fendinger; P. Masscheleyn; L.V. CASTILHO \& J.M. LIM. 2003.The influence of untreated wastewater to aquatic communities in the Balatuin river, the Philippines. Chemosphere, Kidlington, 52: 43-53.

Eklöv, A.G.; L.A. Greenberg; C. Brönmark; P. Larsson \& O. BERgLund. 1998. Response of stream fish to improved water quality: a comparison between the 1960s and 1990s.
Freshwater Biology, Oxford, 40: 771-782.

Fausch, K.D.; L. Lyons; J.R. KarR \& P.L. Angermeier. 1990. Fish communities as indicators of environmental degradation. American Fisheries Society Symposium, Bethesda, 8: 123144.

Gafny, S.; M. Goren \& A. GASITH. 2000. Hábitat condition and fish assemblage structure in a coastal mediterranean stream (Yarqon, Israel) receiving domestic effluent. Hydrobiologia, Dordrecht, 422/423: 319-330.

Gatz JúNIOR. A.J. 1979. Ecological morphology of freshwater stream fishes. Tulane Studies in Zoology and Botany, New Orleans, 21: 91-124.

Gispert, A.V.; E.G. Berthou \& R.M. Amich. 2002. Fish zonation in a Mediterranean stream: Effects of human disturbances. Aquatic Sciences, Basel, 64: 163-170.

Gorman, O. T. \& J.R. KaRR. 1978. Hábitat structure and stream fish communities. Ecology, New York, 59(3): 507-515.

HARRISON, S.; S.J. RosS \& J.H. LAWTON. 1992. Beta diversity on geographic gradients in Britain. Journal of Animal Ecology, Oxford, 61: 151-158.

Honnen, W.; K. Rath; T. Schlegel; A. Schwinger \& D. Frahne. 2001. Chemical analyses of water, sediment and biota in two small streams in southwest Germany. Journal of Aquatic Ecosystem Stress and Recovery, Dordrecht, 8: 195-213.

Hughes, R.M. 1987. Longitudinal changes in fish assemblage and water quality in the Willamette river, Oregon. Transactions of the American Fisheries Society, Bethesda, 116: 196-209.

JACKSON, D.A. 1993. Stopping rules in principal components analyses: a comparison of heuristical and statistical approaches. Ecology, New York, 74: 2204-2214.

Jackson, D.A.; P.R. Peres Neto \& J.D. Olden. 2001. What controls who is where in freshwater fish communities: the roles of biotic, abiotic and spatial factors? Canadian Journal of Fisheries and Aquatic Sciences, Otawa, 58: 157-170.

Jones III, E.B.D.; G.S. Helfman; J.O. Harper \& P.V. Bolstad. 1999. Effects of riparian forest removal on fish assemblages in southern Appalachian streams. Conservation Ecology, Washington, 13 (6): 1454-1465.

KaRR, J.R. \& I.J. Schlosser. 1978.Water resources and the landwater interface. Science, Washington, 201 (4352): 229-234.

KueHne, R.A. 1962. A classification of streams, illustrated by fish distribution in an Eastern Kentucky Creek. Ecology, Washington, 43 (4): 608-614.

LEE, T.R. 2000. Urban water management for better urban life in Latin America. Urban Water, Amsterdam, 2: 71-78.

LeIDY, R.A. \& P.L. Fielder. 1985. Human disturbance and patterns of fish species diversity in the San Francisco Bay drainage, California. Biological Conservation, Kidlington, 33: 247267.

Lemes, E.M. \& V. Garutti. 2002. Ecologia da ictiofauna de um córrego de cabeceira da bacia do alto rio Paraná, Brasil. Iheringia, Série Zoológica, Porto Alegre, 92 (3): 69-78. 
LimbuRG, E.K. \& R.E. SchmidT. 1990. Patterns of fish spawning in Hudson River Tributaries: response to an urban gradient? Ecology, Washington, 71 (4): 1238-1245.

MAGURRAN, A.E. 1988. Ecological diversity and its measurement. Londres, Croom Helm, 179p.

MARGALEF, R. 1963. On certain unifying principles in ecology. The American Naturalist, Chicago, 97 (897): 357-374.

McCune, B. \& M.J. MefFord. 1997. PC-ORD. Multivariate analysis of ecological data, versão 3.12. Oregon, MjM Software Design.

MCDonneLL, M.J. 1997. A paradigm shift. Urban Ecosystems, Dordrecht, 1: 85-86.

Onorato, D.P.; P.A. Angus \& K.R. Marion. 1998. Comparison of a small-mesh seine and a backpack electroshocker for evatuating fish populations in a north-central Alabama stream. North American Journal of Fisheries Management, Bethesda, 18: 361-373.

Orsi, M.L.; E.D. Carvalho \& F. Foresti. 2004. Biologia populacional de Astyanax altiparanae Garutti \& Britski (Teleostei, Characidae) do médio rio Paranapanema, Paraná, Brasil. Revista Brasileira de Zoologia, Curitiba, 21 (2): 207-218.

Osborne, L.L.; D.R. Iredale; F.J. Wrona \& R.W. Davies. 1981. Effects of Chlorinated sewage effluents on fish in the sheep river, Alberta. Transactions of the American Fisheries Society, Bethesda, 110: 536-540.

PAller, M.H. 1994. Relationships between fish assemblage structure and stream order in South Carolina coastal plain streams. Transactions of the American Fisheries Society, Bethesda, 123: 150-161.

Paul, M.J. \& J.L. Meyer. 2001. Streams in the urban landscape. Annual Review of Ecology and Systematics, Palo Alto, 32: 333-365.

Pavanelli, C.S. \& E.P. Caramaschi. 1997. Composition of the ichthyofauna of two small tributaries of the Paraná river, Porto Rico, Paraná State, Brazil. Ichthyological Exploration of Freshwaters, München, 8 (1): 23-31.

Penczak, T.; A.A. Agostinho \& E.K. OKada. 1994. Fish diversity and community structure in two small tributaries of the
Paraná State, Brazil. Hydrobiologia, Dordrecht, 294: 243251.

Pielou, E.C. 1975. Ecological diversity. New York, John Wiley $\&$ Sons, 165p.

ReAsH, R.J. \& T.M. BerRA. 1987. Comparison of Fish Communities in a Clean-water Stream and an Adjacent Polluted Stream. American Midland Naturalist, Notre Dame, 118 (2): 301322.

SAnepar. 2002. Plano de gestão e manejo do manancial do rio Pirapó, Maringá - PR. Maringá, Companhia de Saneamento do Paraná, PADCT/CIAMB; FNDCT/FINEP/CTHIDRO, $53 p$.

SCHLOSSER, I.J. 1982. Fish community structure and function along two hábitat gradients in headwater stream. Ecological Monographs, Washington, 52 (4): 395-414.

SHELDON, A.L. 1968. Species diversity and longitudinal succession in stream fishes. Ecology, Washington, 49 (2) 193-198.

SILVA, C.P.D. 1995. Community structure of in urban and natural streams in the Central Amazon. Amazoniana, Kiel, 8 (3/4): 221-236.

Tramer, E.J. \& P.M. Rogers. 1973. Diversity and Longitudinal Zonation in Fish Populations of Two Streams Entering a Metropolitan Area. American Midland Naturalist, Notre Dame, 90 (2): 366-374.

Turnpenny, A.W.H. \& R. Williams. 1981. Factors affecting the recovery of fish populations in an industrial river. Environmental Pollution, Essex, 26: 39-58.

UIEDA, V.S. 1984. Ocorrência e distribuição dos peixes em um riacho de água doce. Revista Brasileira de Biologia, Rio de Janeiro, 44 (2): 203-213.

Vannote, R.C.; G.W. Minshall; K. Cummins; J.R. Sedell \& C.E. Cushing. 1980. The river continuum concept. Canadian Journal Fisheries and Aquatic Sciences, Otawa, 37: 130137.

ZIPPIN, C. 1958. An evaluation of the removal method of estimating animal populations. Biometrics, Washington, 12: 163-169.

Recebido em 27.XI.2005; aceito em 27.X.2006. 